PRAXIS

ecucativa

Universidad Nacional de La Pampa

Facultad de Ciencias Humanas

Instituto de Ciencias de la Educación

para la investigación interdisciplinaria

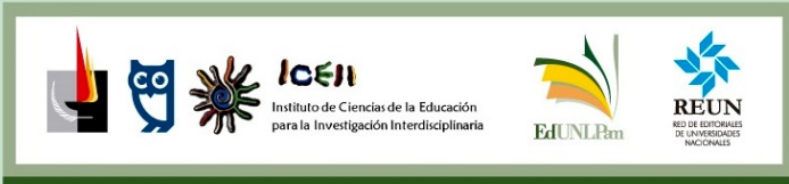

ISSN 2313-934X

SANTA ROSA, LA PAMPA, ARGENTINA

Correo electrónico: iceii@humanas.unlpam.edu.ar

Disponible en https://cerac.unlpam.edu.ar/index.php/praxis

¿Por qué elegir una escuela alternativa?. Artículo de Manuel Morales Valero y Diana Amber Montes. Praxis educativa, Vol. 25, No 3 septiembre - diciembre 2021. E - ISSN 2313-934X. pp. 1-16. https://dx.doi.org/10.19137/praxiseducativa-2021-250308

Esta obra se publica baja Licencia Creative Commons 4.0 Internacional CC BY- NC-SA Atribución, No Comercial, Compartir igual

(c) (1)(2) (2)

\title{
¿Por qué elegir una escuela alternativa?
}

¿Why to choose an alternative school?

¿Por que escolher uma escola alternativa?

\section{Manuel Morales Valero}

Universidad de Málaga, España

mmoralesvalero@uma.es

ORCID0000-0001-6155-2800

\section{Diana Amber Montes}

Universidad de Málaga, España

damber@ujaen.es

ORCID0000-0002-9765-3547

Recibido: 2021- 03-27 | Revisado: 2021-06-10 | Aceptado: 2021-07-13 


\section{Resumen}

Esta investigación pretende identificar y reflexionar sobre los motivos que se encuentran detrás de la elección de un centro de educación alternativo para la etapa de Educación Infantil en España. Para ello, se utiliza una metodología biográfico-narrativa basada en un estudio de caso múltiple compuesto por 24 familias. Así, se identifican cuatro categorías motivacionales: promoción del desarrollo integral de la persona, oposición a la escuela tradicional, búsqueda de la felicidad en la infancia y confianza en los principios pedagógicos. Las familias analizadas valoran especialmente una educación integral adaptada a las necesidades de la infancia más allá de su finalidad instrumental, considerando a la escuela pública como el extremo opuesto a los principios y valores defendidos desde los proyectos alternativos. Se concluye apuntando cómo estas motivaciones, además de tener un sentido pedagógico, responden a una estrategia más amplia de reproducción de la identidad y estilos de vida.

Palabras clave: crisis de la educación, educación de la primera infancia, educación no-formal, teoría de la educación.

\section{Abstract}

This research aims to identify and reflect on the reasons behind the choice of an alternative education centre for the stage of Early Childhood Education in Spain. For this purpose, it is used a biographical-narrative methodology based on a multiple case study made up of 24 families. That way, four major motivational categories are identified: promotion of the integral development of the person, opposition to the traditional school, search for happiness in childhood and trust in the principles pedagogical. Analysed families especially value a comprehensive education beyond its instrumental purpose, they consider public school as the extreme opposite to the principles and values defended from alternative projects. It concludes by pointing out how these motivations, in addition to having a pedagogical sense, respond to a broader strategy to reproduce their identity and lifestyles.

Keywords: crisis of education; early childhood education; non-formal education; educational theory.

\section{Resumo}

Esta investigação pretende identificar e refletir sobre os motivos que se encontram por trás da escolha de um centro de educação alternativo para a Educação da Primeira Infância, na Espanha. Para isso, utiliza-se uma metodologia biográfico-narrativa baseada em um estudo de caso múltiplo composto por 24 famílias. Assim, identificam-se quatro categorias motivacionais: promoção do desenvolvimento integral da pessoa, oposição à escola tradicional, procura da felicidade na infância e confiança nos princípios pedagógicos. As famílias analisadas valoram, especialmente, uma educação integral adaptada às necessidades da infância além da finalidade instrumental, considerando à escola pública como o extremo oposto aos princípios e valores defendidos pelos projetos alternativos. Conclui-se apontando como estas motivações, ademais de ter um sentido pedagógico, respondem a uma estratégia mais ampla de reprodução da identidade e os estilos de vida.

Palavras-chave: crise da educação; educação da primeira infância; educação não-formal; teoria da educação. 


\section{Introducción}

El propósito de este trabajo es el de responder, dentro del ámbito del estudio de caso que nos ocupa, a las siguientes preguntas de investigación: ¿qué motivos se encuentran detrás de la elección de un centro de educación alternativo para la etapa de educación infantil?, ¿qué consecuencias sociales implican?

Si bien la educación infantil es una etapa educativa con identidad propia, su carácter es voluntario, es decir, no existe obligatoriedad por parte de las familias de escolarizar a sus hijos en el tramo de cero a seis años. Se encuentra dividida en dos ciclos diferenciados, de cero a tres y de tres a seis años. Mientras la oferta del primero se encuentra determinada en gran medida por el sector privado (Ministerio de Educación Formación y Profesional, 2018), el segundo está ya plenamente integrado en la red estatal de centros escolares. Con respecto a la tasa de escolarización, se puede hablar de un carácter escalonado y progresivo de cero a tres años alcanzando su saturación a partir de dicha edad (Ministerio de Educación Formación Profesional, 2018; Consejería de Educación y Deporte, 2019). Esta situación administrativa hace que la educación infantil sea más proclive que otras etapas al surgimiento de proyectos de educación alternativa de carácter privado estimulando, a su vez, la demanda de las familias en este sentido.

Entendemos por proyectos de educación alternativa aquellos que, de alguna forma, suponen una reacción crítica al contenido, metodología y, en general, a la institucionalización de la propia educación. Aunque el término pedagogías alternativas puede ser amplio y confuso, se caracteriza por su inspiración en el movimiento de la Escuela Nueva (Díaz, 2019).

Este tipo de proyectos suelen crearse a iniciativa de un conjunto de familias con unas mismas inquietudes sobre la educación, a menudo lideradas por algún padre o madre con formación sobre pedagogías alternativas o, en caso de no existir, por alguna otra persona externa. La mayoría se crean para el segundo ciclo de la etapa de educación infantil debido, como apuntamos, a su no obligatoriedad (Díaz, 2019). Así, mientras su práctica mayoría atiende a esta etapa, solo unos pocos se ocupan de los/as niños/as mayores de seis años, edad en la cual la provisión de educación no homologada no es legal en España. El hecho de que esto sea así es que, si bien muchas familias no tienen ningún problema en escolarizar a sus hijos en centros de educación alternativos, la edad de seis años supone un punto de inflexión en sus decisiones por evitar los problemas burocrático-legales a los que se pudieran enfrentar, incluso aunque la vía desescolarizadora pudiera encajar dentro de la Constitución Española de 1978 (Valle, 2012).

El análisis que aquí proponemos se desarrolla sobre un estudio de caso de uno de estos proyectos alternativos situado en la provincia de Granada (España) cuya identidad no desvelaremos por petición expresa de la dirección del centro para garantizar su anonimato. Se dirige a la educación de la infancia (de cero a seis años) situándose su propuesta desde distintos principios pedagógicos y organizativos (Morales y Amber, 2021) que, a modo general, podríamos enmarcar dentro de las denominadas como pedagogías ligeras (Igelmo y Quiroga, 2018) como continuidad o consecuencia de las denominadas teorías de la desescolarización (Igelmo y Laudo, 2017). 
Aunque lo que nos interesa es conocer los motivos de elección de las familias que deciden apostar por este tipo de centros, estos van a depender de la idiosincrasia de las mismas. En este sentido, una de las variables más influyentes a la hora de hacer efectiva la elección de centro es la clase social, un constructo que mantiene su vigencia como programa de investigación pese a las críticas recibidas (Goldthorpe y Marshall, 2017). Entendemos la clase social más como una posición determinada (consciente o inconscientemente) en base a una identidad y a un estilo de vida (Ball, 2003) que en cuanto a un nivel de renta. Así, nos remitimos al concepto de habitus propuesto por Bourdieu (1991) y su distinción entre capital económico, cultural y social como variables explicativas del orden social (Bourdieu, 2001). Si bien es verdad que el capital económico medido por el nivel de renta es una potente variable explicativa en cuanto a la elección de centro (Fuenmayor et al., 2003), en nuestro caso de estudio, son los capitales cultural y social los que caracterizan en mayor medida a las familias analizadas. Así, además de contar con un amplio capital cultural relacionado con la adopción de una perspectiva crítica de la sociedad, suelen participar en redes sociales donde comparten dicha visión social. El capital cultural incorporado juega un importante papel a la hora de condicionar la elección de centro en tanto su transmisión adquiere una gran importancia para las familias en tanto suponen un gran peso identitario para ellas (Morales y Amber, 2021). Del mismo modo, el capital social funciona especialmente a la hora de construir la fama del proyecto analizado en tanto esta se construye con base en los estilos de vida y modos de pensar de las familias toda vez que se desarrolla en el seno de las redes sociales de los sujetos (Peláez-Paz, 2020).

La investigación se encuadra, por tanto, dentro de dos temáticas contrapuestas: los procesos de crítica a la institución escolar y los procesos de elección de centro educativo en el contexto de cuasimercado de la provincia de Granada (España). Así, su objetivo principal es el de identificar los motivos para optar por un modelo educativo alternativo, así como reflexionar sobre sus consecuencias sociales.

\section{Metodología}

Se ha utilizado un diseño metodológico cualitativo de carácter emergente y flexible, con un enfoque biográfico-narrativo (Bolívar y Domingo, 2019) basado en un estudio de caso múltiple (Stake, 2013). El sujeto de estudio es el grupo en su conjunto en tanto, por medio de los relatos paralelos sobre el eje central de la investigación, las narrativas individuales adquieren un sentido contextualizado (Bolívar, 2012).

La selección muestral se ha llevado a cabo a través de un proceso de bola de nieve (RuizOlabuénaga, 2012), siendo su acceso posibilitado gracias a la colaboración del equipo directivo. La muestra ha quedado determinada por la propia dimensión del estudio de caso quedando conformada finalmente por 24 informantes, nueve padres y 15 madres, donde al menos existe un representante de cada una de las familias con hijos o hijas matriculados en el centro en la fecha del estudio. El 79.17 \% de los informantes dispone de estudios universitarios y más de la mitad, el 58.33 \%, tienen algún tipo de formación pedagógica reglada. 
El instrumento utilizado para la recogida de los datos fue la observación participante durante un periodo de un año y la entrevista en profundidad a las familias partiendo de un guion conversacional flexible que orientó el discurso hacia los aspectos motivaciones de la elección de centro por parte de las familias sin limitarlo, ni coartarlo, permitiendo su fluidez dialógica y su validación dialéctica. El foco central fueron los significados otorgados al fenómeno de estudio, atendiendo a los momentos clave y experiencias que han marcado su decisión (Denzin, 2014). Las entrevistas fueron realizadas durante el segundo trimestre de 2019 y todas ellas han sido transcritas para su posterior codificación y análisis con ayuda de la doceava versión del software QSR NVivo. Para el análisis de la información, se opta por una metodología híbrida tomando aportes de la teoría fundamentada, de la investigación dialógica y del análisis del discurso, desde el marco de la investigación biográfico-narrativa.

La perspectiva polifónica derivada del estudio multicaso, a partir de su análisis horizontal, permitió la detección de leitmotivs presentes en los relatos individuales (Bolívar y Domingo, 2019). Se realizó la codificación del discurso de los informantes mediante un procedimiento inspirado en la Teoría Fundamentada (Hernández, 2014), que permitió la extracción de un sistema de categorías emergente de los relatos de los participantes. El análisis del discurso realizado fue facilitado y estructurado con ayuda de las herramientas de exploración de NVivo, que permitieron la jerarquización de las codificaciones, el contraste de los relatos y el análisis de conglomerados por similitud de codificación mediante el uso del coeficiente de Jaccard.

\section{Resultados}

Las categorías resultantes del análisis de los relatos se organizan en torno a cuatro bloques emergentes que incluyen catorce aspectos motivacionales. El sistema categorial queda representado en la Figura 1, que delimita jerárquicamente los cuatro bloques y las categorías que los conforman, ajustando las dimensiones de cada espacio categorial a la frecuencia de las citas contenidas. 


\section{Figura 1}

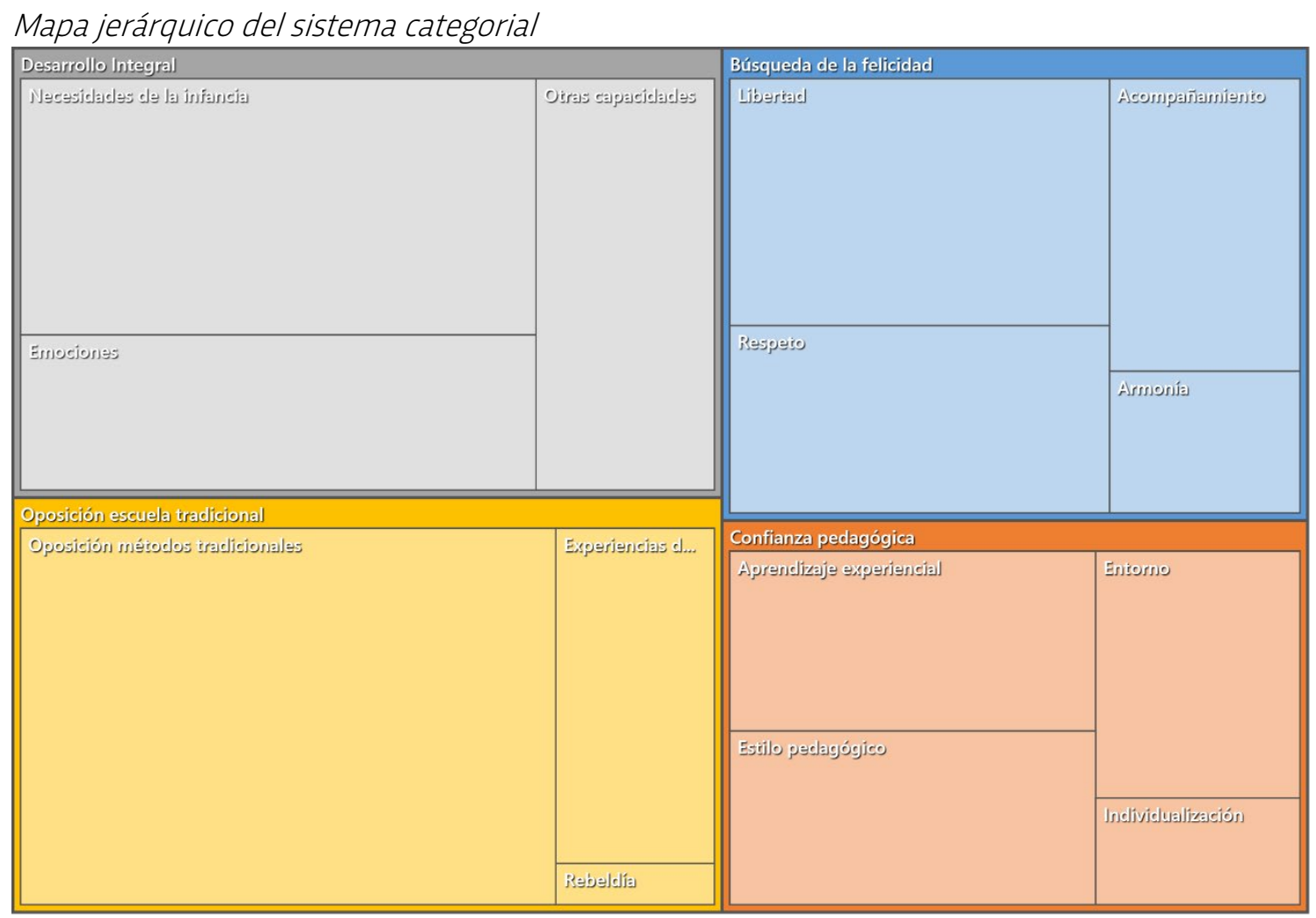

Como se observa en la Figura 1, las motivaciones de los informantes hacia el proyecto educativo en el que se encuentran inmersos se engloban en cuatro tendencias motivacionales esenciales. Aunque todas ellas gozan de una alta presencia en los relatos de los casos, ordenadas por su mayor peso discursivo, estas son: la promoción del desarrollo integral de la persona, la oposición a la escuela tradicional, la búsqueda de la felicidad de la infancia y la confianza en los principios pedagógicos de la escuela alternativa.

La narrativa de estos cuatro bloques motivacionales y sus correlaciones categoriales develadas a partir del análisis de conglomerados por similitud de codificación conforman los cinco apartados en los que se estructuran los resultados de este estudio. A fin de asegurar la confidencialidad de las familias, se utilizan pseudónimos para nombrar a los informantes.

\section{Promoción del desarrollo integral de la persona}

El desarrollo integral de la infancia es una de las preocupaciones de los informantes, que consideran que esta opción pedagógica puede cubrir todas las dimensiones de la persona (afectiva, social, corporal, cognitiva...), alejándose de posturas centradas únicamente en el desarrollo de capacidades intelectuales, que obvian otras facetas del desarrollo humano y apostando por la promoción de una escuela que prepara para la vida en sociedad, en todas sus dimensiones. En palabras de Melia, "creo que una persona no tiene que estar preparada académicamente, sino preparada para la vida" (comunicación personal, 14 de mayo de 2019). Dentro de este bloque 
categorial, se incluyen los relatos que aluden al respeto de las necesidades de la infancia, a la gestión de las emociones como aspecto esencial del desarrollo y a la consideración de otras capacidades (corporales, sociales, etc.), además de las intelectuales.

Para los participantes, es vital adecuar el proceso educativo a las necesidades, ritmos e intereses de los infantes. Conscientes de que "No todos los niños pueden aprender lo mismo a la vez" (Teca, comunicación personal, 16 de abril de 2019), se procura el "Respeto a sus intereses, a su motivación, a sus espacios, a sus tiempos..." (Álamo, comunicación personal, 02 de mayo de 2019). A esta diversidad responden no solo los acompañantes (docentes) con su propia actuación, sino también los distintos espacios y recursos que ofrece el centro, observados durante las visitas a los diferentes escenarios educativos, tanto interiores (zonas de descanso, de juego y movimiento, de lectoescritura, etc.) como exteriores, en un entorno natural.

Valoran especialmente la adecuada gestión de las emociones y el logro de la estabilidad emocional, como base fundamental para el alcance de aprendizajes posteriores. Para ello, manifiestan que los acompañantes ayudan a los infantes a reconocer y expresar sus sentimientos desde el respeto, "dándoles palabras" (Areca, comunicación personal, 21 de junio de 2019) para hacerlo posible. Como apunta Caoba: "No se trata de encontrar al culpable, sino de verbalizar, de ayudar a los niños a expresar qué ha pasado, de ser oídos" (comunicación personal, 22 de mayo de 2019).

Las familias participantes desean que sus hijos e hijas puedan descubrirse ellos mismos, optimizar sus potencialidades, sin ser coartados o dirigidos coercitivamente, tan solo siendo acompañados en la dirección que ellos mismos van marcando. Con esa convicción, Palma alega que lo más importe que enseña esta escuela es "a saber quién eres tú" (comunicación personal, 18 de abril de 2019).

\section{Oposición a la escuela tradicional}

La segunda motivación más aludida por los informantes tiene un marcado matiz reivindicativo, centrándose en la oposición a la escuela tradicional, a sus métodos y principios pedagógicos y a los valores sociales de sumisión, acatamiento y uniformidad que esta representa. Esta oposición detectada en el discurso de los informantes, en muchas ocasiones, es generada por experiencias desencadenantes vividas en primera persona por los propios participantes o a través de sus descendientes, que también quedan recogidas en su discurso. Estas vivencias propias incitan a la rebeldía o insumisión social, expresada a través de su elección pedagógica.

Critican la alienación a la que se somete a los discentes desde la enseñanza tradicional, que asocian a la escuela pública: "no creo en el sistema de enseñanza tradicional que se da en la escuela pública" (Secuoya, comunicación personal, 06 de abril de 2019). Consideran que esta tiene el objetivo de crear ciudadanos sumisos, obedientes y productivos, mediante su sometimiento y el uso de métodos de uniformización. Así, Morinda asegura que la enseñanza tradicional "coarta la libertad, coarta la crecida, coarta pues, el pensamiento divergente; al final todos piensan lo mismo" (comunicación personal, 18 de junio de 2019). Consecuentemente, alegan que el sistema educativo 
tradicional siega las individualidades y no permite que afloren las potencialidades intrínsecas de cada persona. Apoyan sus palabras en su propia vivencia académica en la escuela pública, señalando vestigios en su personalidad, atribuidos al método de enseñanza:

Veo que mi capacidad de investigar, de desarrollar proyectos por mí mismo está muy mermada, estoy acostumbrado a que me lo den todo hecho, a elegir entre opciones, no tengo esa capacidad creativa y creo que en parte se lo debo a eso, a que no es lo que me han fomentado en la escuela. (Laurel, comunicación personal, 16 de mayo de 2019)

Los informantes se oponen al magistrocentrismo que consideran aún imperante en la escuela pública, como huella viva y persistente de la enseñanza tradicional en la actualidad. Conciben al docente tradicional como una figura autoritaria, cuya palabra es la norma y representa el saber y a la que hay que respetar y temer. Aunque en muchas ocasiones reconocen que basan sus alegaciones en su propia experiencia personal como discentes y en los vívidos recuerdos y sentimientos que aún asocian a ella: "Como yo lo he vivido, es un sistema de adoctrinamiento, enseñan a que hay una autoridad, a la que hay que obedecer, además, posee la verdad, y te adaptas y te sometes o estás excluido y marginado de alguna forma" (Laurel, comunicación personal, 16 de mayo de 2019).

Los informantes afirman que los métodos tradicionales inhiben el diálogo, coartan la expresión infantil, generando una relación vertical entre docente y discente, basada en el verbalismo adulto, que promueve la pasividad y receptividad del alumnado: "En la escuela tradicional el profesor habla, te cuenta, te dice y tú vas copiando o vas escuchando simplemente" (Sabina, comunicación personal, 10 de junio de 2019). Los informantes hablan de pasividad, no solo dialógica, sino también física, de limitación de movimientos, alegando que los niños y niñas necesitan actividad física: "son muchas horas para unos niños sentados" (Haya, comunicación personal, 09 de junio de 2019). Además, descartan posturas memoristas y enciclopedistas, apostando por el uso de materiales manipulativos, recursos naturales y el juego: "Estudiar la fotosíntesis en un libro o en una pantalla digital, teniendo el patio lleno de árboles... No lo entiendo" (Nogal, comunicación personal, 12 de abril de 2019).

Describen a la escuela pública como "arcaica" u "obsoleta", asegurando que existe una falta de correlación entre la sociedad y la escuela, evidenciada no solo en sus contenidos y métodos, centrados en lo académico, sino también en su restricción de acceso a las familias y a otros agentes sociales.

Su motivación principal es alejarse de todo aquello que asocian a la escuela pública y que rechazan, como apuntamos, en muchas ocasiones por sus vivencias personales. Sus sentimientos propios arraigados a sus recuerdos escolares marcan su decisión actual, como reconoce Olivo: "A mi pareja y a mí, lo que nos pasa es que tenemos una historia con la escuela bastante traumática, con la escuela pública" (comunicación personal, 11 de mayo de 2019) y ratifica Koa al argumentar que evitan la escuela pública "por miedo a que ellos, a que tus hijos, sufran lo mismo que sufriste tú, por evitar la misma experiencia de vida que no te gustó a ti" (comunicación personal, 27 de abril de 2019). Desde el sentimiento de rebelión a la opresión social, con tintes reivindicativos, Caoba añade 
que evita la escuela pública por "un poco de desobediencia, se puede decir, pero desde el sentimiento de justicia, de evitarle a mi hijo que vivencie lo que yo he tenido que vivir, que es buscarme a mí misma en la edad adulta" (comunicación personal, 22 de mayo de 2019).

\section{Búsqueda de la felicidad de la infancia}

Más allá de los logros académicos, los participantes buscan la felicidad que consideran que este estilo educativo puede aportarle a sus hijos e hijas, la armonía con la sociedad, con la naturaleza y con ellos mismos, a través del respeto y la libertad. Armonía, libertad y respecto en un proceso guiado, mediante el acompañamiento adulto durante su desarrollo, son los leitmotivs que integran este bloque motivacional, caracterizado por el deseo del logro de la felicidad de la infancia. Así, Encina asegura que los contenidos académicos no le inquietan "porque sé que eso va a ir llegando, a mí lo que me preocupa es que no sea feliz" (comunicación personal, 16 de abril de 2019).

Se busca un desarrollo armónico de los infantes, considerando sus ritmos e intereses, educando desde el respeto y para el respeto "hacia los otros compañeros y hacia el entorno y hacia las otras personas que allí están, pero son libres de decidir lo que quieren hacer" (Acacia, comunicación personal, 24 de mayo de 2019). El respecto a los intereses y motivaciones de la infancia es altamente valorado por los informantes, que llegan a definirlo como "un regalo para toda su vida, cosa que la mayoría de nosotros no hemos tenido" (Kentia, comunicación personal, 17 de abril de 2019).

Junto al respeto, la libertad es uno de los pilares fundamentales del estilo educativo por el que optan los informantes: "Me gusta la libertad, me gusta que los niños aprendan de forma libre, me gusta que sean ellos los que deciden qué quieren hacer y qué no" (Nogal, comunicación personal, 12 de abril de 2019). La apuesta por la libertad queda patente no solo en el discurso de los informantes, sino también en las propuestas educativas que se ofrecen, en la distribución de los espacios del centro y en los aspectos organizativos del proyecto, como el horario flexible, señalado por la directora y muy valorado por las familias, como se aprecia en las palabras de Olmo: "no hay una estructura rígida mediante unos horarios rígidos, sino que estamos en un proyecto, en el cual hay apertura a la hora de decidir" (comunicación personal, 28 de junio de 2019).

Se produce un acompañamiento no solo por parte de los docentes (acompañantes), sino también por parte de las familias, que pueden visitar los espacios del centro y permanecer al lado de sus hijos o hijas el tiempo necesario para que se produzca una progresiva adaptación al nuevo medio, evitando la frustración generada por la separación de la figura de apego. Así describe Roble el periodo de adaptación de su hija:

Yo estuve 3 meses con mi hija, hasta que se adaptó a quedarse sola. Al principio ella no se despegaba de mí, luego le encantaba ir, pero si iba yo con ella. Tenía que tener la seguridad de que me veía, que estaba yo por ahí (comunicación personal, 14 de abril de 2019).

Esta posibilidad de acceso al centro educativo es una oportunidad muy valorada por los informantes que optan por esta propuesta pedagógica, asegurando que el menor "no pasa por un proceso traumático de adaptación a la escuela, porque están sus padres continuamente 
acompañándole" (Olivo, comunicación personal, 11 de mayo de 2019), lo que contribuye a su felicidad y bienestar.

\section{Confianza en los principios pedagógicos de la escuela alternativa}

El cuarto bloque motivacional hace alusión a la firme convicción de las familias de que el estilo pedagógico, métodos y principios que, aun con matices distintivos, caracterizan a la educación alternativa son beneficiosos para el adecuado desarrollo infantil. Características que ven reflejadas en el proyecto educativo en el que participan, cuya confianza e interés por su línea pedagógica hace a muchos informantes incluso desplazarse desde otros municipios de la provincia para llevar diariamente a sus vástagos al centro o para participar en sus reuniones y propuestas comunitarias.

De este modo, muchos informantes, especialmente aquellos con formación pedagógica reglada, alegan que su motivación principal para la elección del centro fue su estilo pedagógico, pues apuestan por una educación activa y alternativa, que confiera el rol principal al discente y le conceda libertad para desarrollarse de forma progresiva y natural, sin apremios, ni exigencias.

Defienden el uso de la observación directa y la experimentación activa como forma de aprendizaje, que dote de sentido a los contenidos, concediendo especial importancia al entorno y al contacto directo con la naturaleza. Desde este planteamiento, cuando Sabina explica las razones por las que escogió este proyecto, argumenta: "Les enseñan a los niños a coger la aceituna, a pisar la uva, a cuidar el huerto... Le inculcan el amor a la naturaleza. Ese tipo de cosas son las que han hecho que esté allí" (Sabina, comunicación personal, 08 de mayo de 2019).

El entorno natural en el que se ubica el centro, la vegetación, el huerto, el agua, el espacio natural que lo envuelve, es una de las características más destacadas del proyecto, que lo distingue de los centros públicos por los recursos y medios naturales que ofrece. Esto es un gran reclamo para las familias entrevistadas, pues como asegura Ébano: "no todas las escuelas tienen ahí un espacio natural alrededor" (comunicación personal, 19 de abril de 2019) que Álamo describe como "una finca grande, llena de naturaleza, de vegetación, con columpios, zonas de escalada, de movimiento e incluso piscinas" (comunicación personal, 02 de mayo de 2019).

La baja ratio (cinco o seis niños por acompañante adulto, como se pudo observar durante las visitas al centro) es otra de las razones principales que motivan la elección de este proyecto educativo por parte de las familias, pues permite una atención individualizada, que respeta la diversidad y atiende a las casuísticas personales. Son muchos los progenitores que hacen alusión a esta cuestión entre sus razones, alegando que "no todos los niños pueden aprender lo mismo a la vez, no es posible, y con ratios tan altas [en la escuela pública], se tiende a unificar" (Teca, comunicación personal, 16 de abril de 2019). La atención individualizada favorece a su vez el diálogo, no solo entre los pequeños, sino también entre docentes y discentes, desde una horizontalidad basada en el respeto, una relación "de tú a tú", como la describe Sabina (comunicación personal, 08 de mayo de 2019). 


\section{Correlaciones entre categorías motivacionales}

Una vez analizadas las cuatro tendencias motivacionales emergentes, el análisis de conglomerados por similitud de codificación arrojó información sobre las correlaciones existentes entre las categorías que conforman los cuatro bloques categoriales. La Figura 2, resultante de este análisis, mostró las relaciones que presentan coeficientes de Jaccard más elevados, tras ajustar el límite inferior del índice de similitudes a 0,6.

\section{Figura 2}

Diagrama grafo circular de elementos conglomerados por similitud de codificación

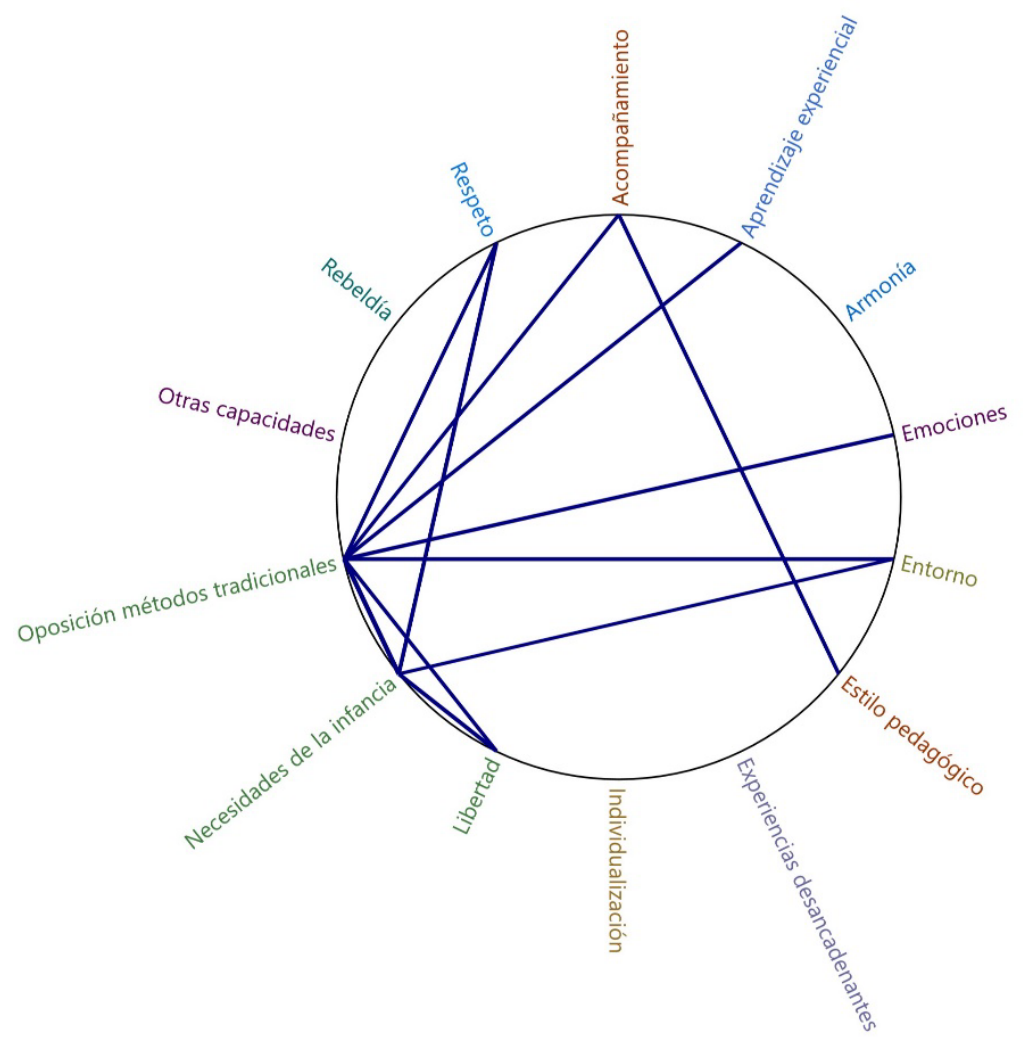

La correlación categorial más fuerte se estableció entre las categorías "oposición a los métodos tradicionales" y "necesidades de la infancia" con un coeficiente de Jaccard de 0,83. Esto señala la creencia por parte de los informantes de que los métodos de la enseñanza tradicional no responden adecuadamente a las necesidades de la infancia. La categoría denominada "necesidades de la infancia" presenta asimismo una fuerte relación con "libertad" y "respeto" (no solo como señas de identidad del proyecto, recogidas en los documentos del centro, sino también como valores imprescindibles para el desarrollo infantil) y con "entorno", como mediador necesario para el logro de los propósitos educativos.

La lectura de la Figura 2 descubre que el eje central discursivo es la oposición a los métodos tradicionales manifiesta por parte de los informantes, que consideran que comparativamente este 
proyecto educativo es "el mejor de lo que hay" (Morinda, comunicación personal, 18 de junio de 2019). El hilo argumentativo de los informantes gira en torno al contraste de las virtudes del proyecto en el que participan con los métodos y estrategias que perciben que implementa la escuela pública. Así, la categoría "oposición a los métodos tradicionales" muestra correlación no solo con las "necesidades de la infancia", sino también con libertad y respeto, con acompañamiento, con aprendizaje experiencial, con emociones y con entorno. Todos ellos elementos clave del proyecto alternativo en el que se inscriben los informantes, que no han hallado en la enseñanza pública y a la que se remiten constantemente para ilustrar y ejemplificar sus argumentos. De este modo, la escuela tradicional y la oposición a esta es un referente discursivo recurrente en los relatos familiares.

\section{Discusión y conclusiones}

Con el análisis presentado, hemos identificado, tal y como nos planteábamos, los motivos declarados por las familias para guiar su elección de un proyecto de educación alternativo en educación infantil. Si bien los resultados de cualquier estudio pueden ser interpretados desde distintos puntos de vista, a continuación, destacamos las principales conclusiones que podemos extraer con base en nuestro periodo de observación directa en el campo de estudio.

\section{La importancia de una finalidad no instrumental de la educación}

En primer lugar, destacamos el hecho de que se valore la adopción de una perspectiva más equilibrada de los aprendizajes. Esteve (2010) apuntaba a la importancia de este equilibrio para considerar un aprendizaje como educativo. Del mismo modo, al establecer la disyuntiva entre el desarrollo de capacidades intelectuales vs. "preparación para la vida" se está situando la finalidad de la educación más allá de un orden instrumental (Bernstein, 1988) o, por lo menos, no en el sentido de la adquisición de competencias profesionales para el empleo.

Asimismo, en aras de dicho equilibrio, se le concede una especial importancia a la gestión de las emociones, un pilar de la educación que se considera desatendido en la escuela pública, aunque en los últimos años cada vez se le haya prestado más atención en la formación del profesorado (Escolar et al., 2017; Ortega, 2019) pudiendo considerarse incluso como una línea de investigación emergente (García et al., 2019).

\section{Adaptación a las necesidades y desarrollo de la infancia, más allá de la función de custodia}

El análisis correlacional nos ha mostrado cómo las familias consideran que los métodos de enseñanza tradicionales (basados en la transmisión de la información) no responden a las necesidades propias de la infancia como etapa evolutiva con identidad propia. Efectivamente, es una seña de identidad de este tipo de proyectos el situar al alumnado en el centro del proceso de enseñanza-aprendizaje, es decir, el seguir una línea paidocéntrica. Así, se entiende que, para 
alcanzar un aprendizaje equilibrado en el sentido señalado, los recursos didácticos puestos en juego deben ser acordes a dicha finalidad. En otro lugar, hemos descrito detenidamente los principios pedagógicos y valores organizativos de este tipo de proyectos (Morales y Amber, 2021). En concreto, en esta investigación, las necesidades de la infancia se relacionan especialmente con libertad (atención a métodos pedagógicos no directivos), respeto en la relación pedagógica y cuidado del entorno para que sea enriquecedor en sí mismo. Al fin y al cabo, de lo que se está hablando es de la importancia de utilizar una metodología activa que, por otro lado, se entiende que no sería posible sin una baja ratio.

Todo este entramado discursivo nos permite afirmar que la función otorgada a la escuela no es la tan denostada custodia, sino precisamente la apuesta por un desarrollo integral. De este modo, no es de extrañar que los argumentos utilizados en esta categoría encuentren cierta correlación con las categorías generales búsqueda de la felicidad y confianza pedagógica. Por otro lado, el hecho de que la función de custodia sea irrelevante da cuenta de la centralidad que ocupa la educación, tal y como se ha descrito, para estas familias. Así, la práctica mayoría no tiene ningún problema en sacrificar tiempo, esfuerzo y unos recursos económicos muchas veces limitados para que sus hijos/as asistan al proyecto. De hecho, muchas realizan importantes desplazamientos diarios habiendo cambiado incluso su lugar de residencia para encontrarse más próximas al proyecto. Por consiguiente, es significativo el enorme esfuerzo puesto en juego en pro de la transmisión cultural de sus estilos y modos de vida, del orden expresivo en términos de Bernstein (1998). Además, se podría argüir, tentativamente, que estos discursos tratan de proteger a la infancia de los males que se achaca a la escolarización en el sistema educativo formal.

\section{La escuela pública como la "vieja escuela"}

Efectivamente, el eje central discursivo ha sido la oposición a los métodos tradicionales y estos, a su vez, se presentan como una característica propia de la escuela ordinaria. Podríamos decir que todo el discurso paralelo o en contra de la escolarización obligatoria se construye de forma dicotómica describiéndola como la antítesis de los valores que se intentan defender desde los proyectos alternativos. Así, dicha dicotomía, representada por la oposición entre el desarrollo de capacidades intelectuales vs. preparación para la vida, conlleva una fuerte oposición a la escolarización formal que tachan de plantear unas finalidades excesivamente academicistas y unos métodos que no suelen ser respetuosos con las necesidades del alumnado (magistrocentrismo). Es decir, encierra una reacción de rechazo a una educación, muchas veces fundamentada en experiencias personales.

No obstante, a esta postura, se le pueden plantear algunas objeciones. En primer lugar, desde la aparición del discurso de las competencias la escuela pública ha tomado una deriva más paidocéntrica que magistrocéntrica en múltiples sentidos (Mir y Ferrer, 2014; Alsina, 2013). Cosa bien distinta es que algunos docentes, amparados en su libertad de cátedra, sigan sosteniendo en sus aulas métodos considerados como más tradicionales. En segundo lugar, nos parece que las 
capacidades intelectuales no tienen por qué estar necesariamente reñidas con la preparación para la vida, como han postulado algunos autores (Fernández et al., 2017).

En resumidas cuentas, con base en estos argumentos, podríamos finalizar concluyendo que los motivos de elección de un proyecto alternativo como el analizado responden no solo a criterios estrictamente pedagógicos, sino, sobre todo, a una estrategia familiar para reproducir su identidad de clase y estilos de vida, por otro lado, muy loables en tanto se basan en una versión crítica y comprometida por unas relaciones sociales más justas. No obstante, esta visión de la educación como promotora del cambio social pudiera verse socavada en tanto dichas estrategias basadas en la fama o reputación del mismo, con base en sus lógicas, pueden reducir la diversidad presente en las aulas, dificultando, por tanto, el diálogo intercultural. Así, algunas investigaciones apuntan a la necesidad de limitar, desde la política educativa, estas estrategias de elección de centro precisamente para evitar estas situaciones (Reinoso y Grau, 2008).

Se podría señalar, como principal limitación de esta investigación su escasa representatividad y, por tanto, generalización. En este sentido, hemos tratado de buscar patrones de significado estando más interesados en la generalización vertical de sus conclusiones que en la horizontal, es decir, en identificar "mecanismos, procesos y lógicas que pueden encontrarse en cualquier lugar" (Lorenz, 2017, p. 31). Así, esta posible limitación podría quedar superada por la capacidad de los resultados obtenidos para favorecer la comparación con estudios similares, si bien es cierto que el acceso al campo a este tipo de proyectos no suele ser fácil. Como futura línea de investigación, sería interesante analizar con mucho más detalle la identidad de las familias, su estilo de vida y el grado en que ostentan los distintos tipos de capital señalado (económico, cultural y social) en su configuración debido a la importancia que estas características tienen para la elección de centro educativo.

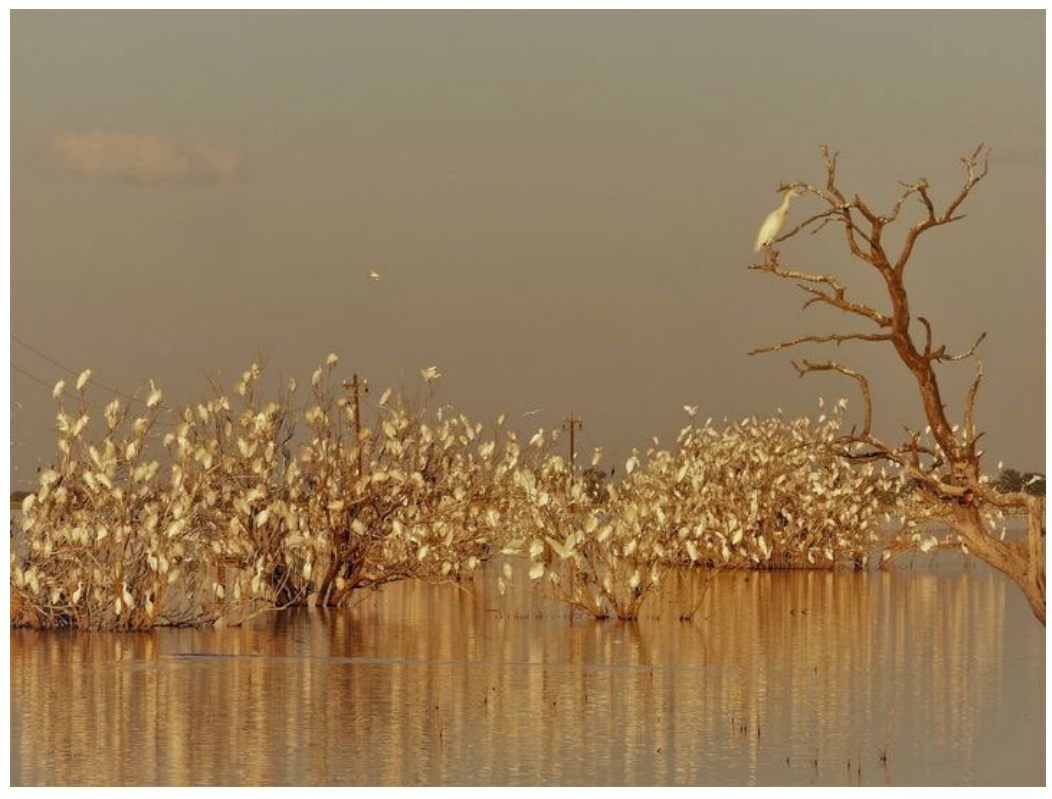

Sin título, fotografía. Andrea Talone 


\section{Bibliografía}

Alsina, A. (2013). Un modelo realista para el desarrollo profesional en la formación inicial de maestros de educación infantil. Revista Electrónica Interuniversitaria de Formación del Profesorado, 16(2), 27-37. http://dx.doi.org/10.6018/reifop.16.2.180761

Ball, S. J. (2003). Class Strategies and the Education Market. The Middle Classes and Social Advantage. Routledge Falmer.

Bernstein, B. (1988). Clases, Códigos y Control. Hacia una teoría de las transmisiones educativas (Vol. II). Akal. Bolívar, A. y Domingo, J. (2019). La investigación (auto)biográfica y narrativa en educación. Octaedro.

Bolívar, A. (2012). Metodología de la investigación biográfico-narrativa: recogida y análisis de datos. En M. C. Passeggi y M. H. Abrahao (Eds.), Dimensões epistemológicas e metodológicas da pesquisa (auto) biográfica (pp. 79-109). Editoria da PUCRS.

Bourdieu, P. (1991). El Sentido Práctico. Taurus.

Bourdieu, P. (2001). Poder, Derecho y Clases Sociales. Desclée de Brouwer.

Consejería de Educación y Deporte. (2019). Sistema andaluz de indicadores de la educación. Recuperado de: https://www.juntadeandalucia.es/organismos/educacionydeporte/servicios/estadistica-

cartografia/actividad/detalle/175109/175571.html (Consultado el 04/01/2020).

Denzin, N. K. (2014). Interpretive autoethnography. Sage Publications.

Díaz, M. P. (2019). Panorama actual de las pedagogías alternativas en España. Papeles salmantinos de educación, (23), 247-281.

Escolar, M., de la Torre, T., Huelmo, J. y Palmero, C. (2017). Educación emocional en los profesores de Educación Infantil: aspecto clave en el desempeño docente. Contextos Educativos. Revista de Educación, O(20), 113-125. https://doi.org/10.18172/con.2994

Esteve, J. M. (2010). Educar: un compromiso con la memoria. Octaedro.

Fernández, C., García, O. y Galindo, E. (2017). Escuela o barbarie: entre el neoliberalismo salvaje y el delirio de la izquierda. AKAL.

Fuenmayor, A., Granell, R. y Villareal, E. (2003). Determinantes de la elección de centro educativo por parte de los padres. Estudios de Economía Aplicada, 27(2),377-389.

García, l., Gallardo, J. A. y López, F. (2019). La inteligencia emocional y la educación emocional en la escuela: un estado de la cuestión a través del análisis bibliométrico de la producción científica en Scopus (2015-2019). En J. A. Marín (Ed.), Inclusión, Tecnología y Sociedad: investigación e innovación en educación (pp. 220-231). Dykinson.

Goldthorpe, J. H. y Marshall, G. (2017). El prometedor futuro del análisis de clase: Una respuesta a las críticas recientes. Revista de Sociología, 32(1), 106-128. https://doi.org/10.5354/0719-529x.2017.47888

Hernández, M. (2014). La investigación cualitativa a través de entrevistas: su análisis mediante la teoría fundamentada. Cuestiones Pedagógicas, 23, 187-210.

Igelmo, J. y Laudo, X. (2017). Las teorías de la desescolarización y su continuidad en la pedagogía líquida del siglo XXI. Educación XX1, 20(1), 37-56. https://doi.org/10.5944/educXX1.11465

Igelmo, J. y Quiroga, P. (2018). La pedagogía ligera en tiempos hipermodernos: el homeschooling, las escuelas Waldors y la nueva pedagogía ignaciana. Teoría de la educación, 30(1), 75-94. http://doi.org/10.14201/teoredu3017594

Lorenz, M. (2017). La policía como objeto de estudio: Entrevista con Didier Fassin. Nueva Sociedad, (268), 23. Recuperado de https://nuso.org/media/articles/downloads/TG_Lorenz_268.pdf (Consultado el 15/12/2019). 
Ministerio de Educación y Formación Profesional (MEFP). (2018). Datos y cifras. Curso escolar 2018/2019. https://sede.educacion.gob.es/publiventa/datos-y-cifras-curso-escolar-20182019/ensenanza-

estadisticas/22495

Mir, M. L. y Ferrer, M. (2014). Aproximación a la situación actual de la formación del profesorado de educación infantil. Revista Electrónica Interuniversitaria de Formación del Profesorado, 17(2), 235-255. http://doi.org/10.6018/reifop.17.2. 181441

Morales, M. y Amber, D. (2021). Implicaciones pedagógicas y organizativas de un proyecto de educación alternativo para la etapa de educación infantil en España. Educatio Siglo XXI.

Ortega, M. C. (2019). La formación en competencias socioemocionales: nueva praxis para la mejora en la relación educativa. En J. Vera (Coord.), Formar para transformar. Cambio social y profesiones educativas (pp. 293-296). GEU

Peláez-Paz, C. (2020). La influencia de la fama de las escuelas en la elección de centro escolar de las familias: un análisis etnográfico. Teoría de la Educación, 32(2), 131-155. http://doi.org/10.14201/teri.22394

Reinoso, A. O. y Grau, E. S. C. (2008). Las familias de clase media y elección de centro: el orden instrumental como condición necesaria pero no suficiente. Profesorado. Revista de Currículum y Formación de Profesorado, 12(2).

Ruiz-Olabuénaga, J. I. (2012). Teoría y práctica de la investigación cualitativa. Deusto.

Stake, R. E. (2013). Multiple Case Study Analysis. Guilford Press.

Valle, J. E. (2012). Enseñar en casa o en la escuela: La doctrina legal sobre el homeschooling en España. Perfiles educativos, 34(138), 167-182. 